\title{
Dynamic Modeling of the Brushless Slip Power Recovery Homopolar Machine
}

\author{
Elhussien Abbas Mahmoud \\ Ain Shams University \\ Corresponding author: elhussien.abbas@gmail.com
}

\begin{abstract}
The brushless slip power recovery Homopolar machine (BSPRHM) is expected to be the dominant wind energy generator. The BSPRHM is one of few machines that are able to provide output voltage of constant frequency regardless of the shaft speed. The field current frequency is the control parameter which helps regulating the output voltage. The inductance matrix is the base stone of the classical machine modeling. As the inductance matrix of the BSPRHM has not been yet identified in any of the published lectures, this work aims to derive the mathematical expression for the BSPRHM inductance matrix. Then a prototype of the BSPRHM is built and the values of its inductance matrix elements are determined through practical experiment. Finally, the evaluated inductance matrix is verified by utilized it together with the classical machine mathematical model and a simulation software to estimate one of the variable in the relation between the BSPRHM armature frequency, field frequency and rotor speed while the other two variables are known. A comparison is carried out among the theoretical, simulation and practical results to prove the accuracy of the proposed BSPRHM inductance matrix.
\end{abstract}

Key words: Homopolar, Slip power recovery, Wind energy, Modelling

\section{1- Art Back Ground}

Nowadays, renewable energy resources are the hope to meet the increasing universe power demands. Wind turbine is an effective example. A wind turbine running on fixed speed to keep the alternator output frequency constant suffers from losing the maximum power operating point. Variable speed wind turbines provide the possibility of tracking the maximum power point but on the expense of variable alternator output voltage. Huge power electronics are required to convert the variable frequency voltage to constant frequency voltage. Later on a race starts to develop electric generators that regulate the output frequency against the variable rotor speed. One option is the doubly fed induction machine but its slip ring is an issue [1]. The brushless doubly fed generators takes over the show but still its rotor losses and design complicity pull it back in the race [2]. The next option is the brushless doubly fed reluctance generator which eliminates the rotor losses, slip rings and rotor complicity but with poor dynamic performance [3]. Reference [4] offers a new machine (BSPRHM) that has the same advantages of the brushless doubly fed reluctance machine and eliminates its disadvantages. Equation (1) states the condition of achieving constant output voltage from the BSPRHM. 
$f_{a}=P f_{r}-f_{f}$

Where:

$f_{a}$ armature current frequency

$f_{f}$ field current frequency

$f_{r}$ rotor shaft mechanical frequency

$P$ number of pole pairs

. Still a lot of work has to be done in order to correctly evaluate the BSPRHM performance. This paper takes the first step in the long way by offering the derivation of the BSPRHM inductance matrix.

\section{2- Inductance Matrix}

The inductance matrix is essential for electric machines classical modeling. The target of the current section is to derive the inductance matrix of the BSPRHM. The starting point is the identification of the flux paths through the machine body. Consider a two pole machine where half of its winding end turns folded upward and the other half folded downward. If the current flows in the direction shown in fig. 1, the flux will mainly pass radially. Due to the existence of a closed low reluctance magnetic pass in the axial direction, part of the flux prefers the axial branch.

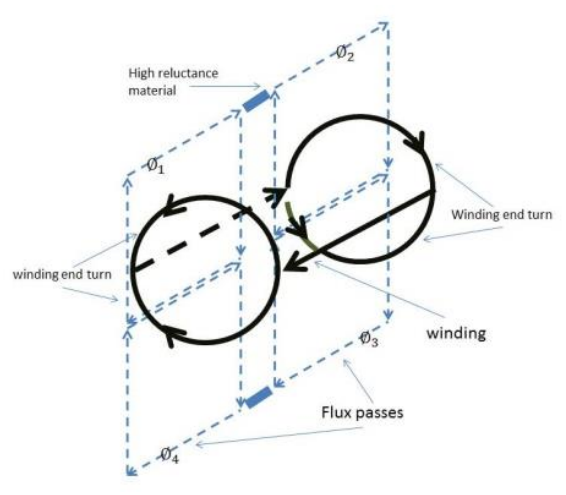

Fig.1 : Flux passes within the BSPRHM

Typically the reluctance of the rotor and yoke iron is negligibly close to zero. The magnetic field intensity drops solely upon the air gaps. The flux paths within the BSPRHM have four air gaps, two in the radial direction and two in the axial direction. The construction shown in fig. 1 is equivalent to the magnetic circuit posted in fig. 2. It could be noticed that each radial air gap is modeled by two semi-parallel reluctances due to the high reluctance material separator embedded in the yoke. The phrase semi-parallel means that they are not parallel but could be parallel in the absence of the separator. The separator could be a relatively large piece of non-magnetic material. 


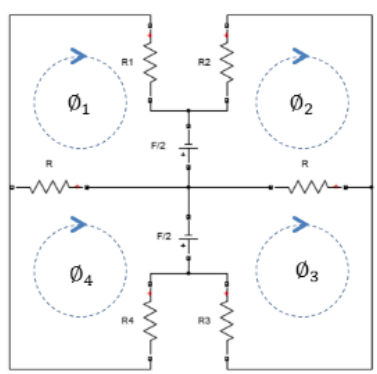

Fig. 2: Magnetic circuit of the BSPRHM

The magneto-motive force is modeled as two parts, one part located before the axial branching while the other part is after the branching. Straight forward application of Kirchhoff laws on the circuit in hand leads to equations (2-5).

$\emptyset_{1}=\frac{F}{2}\left(\frac{2 R+R_{4}}{R R_{1}+R R_{4}+R_{1} R_{4}}\right)$

$\emptyset_{2}=-\frac{F}{2}\left(\frac{2 R+R_{3}}{R R_{2}+R R_{3}+R_{2} R_{3}}\right)$

$\emptyset_{3}=-\frac{F}{2}\left(\frac{2 R+R_{2}}{R R_{2}+R R_{3}+R_{2} R_{3}}\right)$

$\emptyset_{4}=\frac{F}{2}\left(\frac{2 R+R_{1}}{R R_{1}+R R_{4}+R_{1} R_{4}}\right)$

Where: $\mathrm{F}$ is the magneto-motive force.

Fig. 3 provides the left hand side view of a two poles BSPRHM.

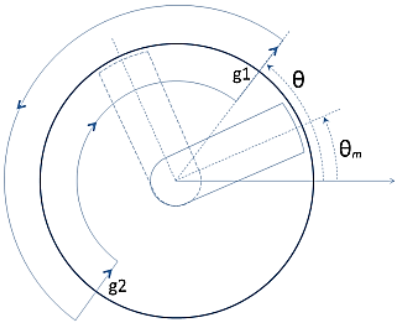

Fig. 3: Left hand side view of the BSPRHM

Each pole occupies only half of the effective longitudinal length of the machine while the other pole occupies the remaining half. The two poles are shifted by $\pi / 2$ electrical degree in space. The value of the radial air gap reluctance varies according to the rotor position where the location of the left hand side pole affects the value of $R_{1}$ and $R_{4}$ while the location of the right hand pole affects the value of $R_{2}$ and $R_{3}$. Given that the width of each pole is $2 \rho$ degree, the radial reluctances at an arbitrary angle $\theta$ could be stated as given in the first four rows in table (1).

The position of the left hand side pole with respect to the horizontal axis is selected to 
be the rotor position $\left(\theta_{m}\right)$. The reluctances values are substituted in equations (2-5) to calculate the flux in each circuit loop. The fluxes in concern are the fluxes crossing the air gap from the stator to the rotor which can be directly evaluated from the loops fluxes as given in table (1).

The fluxes waveform stated in table (1) depends on the value of the MMF. The majority of electrical machines have near-sinusoidal winding. The most famous type of winding has two coils started in two adjacent slots as shown in fig. 4. The generated MMF at an arbitrary angle $\theta$ is evaluated by considering all the ampere turns enclosed by the closed contour shown in fig.3.

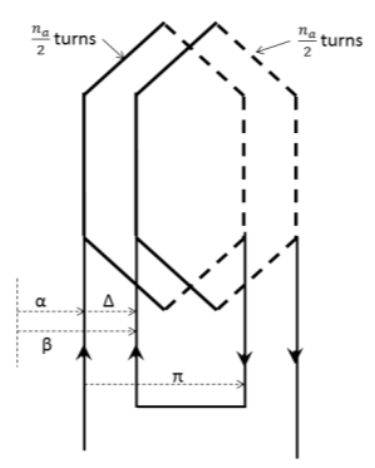

Fig. 4: near sinusoidal winding.

Equation (6) states the MMF of a dual coil winding.

$$
\begin{aligned}
& F(\theta) \\
& =\left\{\begin{array}{cc}
0 & \quad \alpha<\theta<\beta \\
\frac{n_{a} i}{2} & \quad \beta<\theta<\alpha+\pi \\
0 & \alpha+\pi<\theta<\beta+\pi \\
\frac{-n_{a} i}{2} & \beta+\pi<\theta<\alpha+2 \pi \\
\text { Where : } \\
\frac{n_{a}}{2} & \text { is the number of turns for each coil. } \\
\mathrm{i} & \text { is the current carried by the coil. } \\
\alpha & \text { is the starting angle of the first coil. } \\
\beta & \text { is the starting angle of the second coil. }
\end{array}\right.
\end{aligned}
$$

Table 1: Reluctances of the BSPRHM magnetic circuit

\begin{tabular}{|c|c|c|c|c|c|c|c|c|}
\hline$\theta-\theta_{m}$ & $-\rho \rightarrow \rho$ & $\rho \rightarrow \frac{\pi}{2}-\rho$ & $\frac{\pi}{2}-\rho \rightarrow \frac{\pi}{2}+\rho$ & $\frac{\pi}{2}-\rho \rightarrow \pi-\rho$ & $\pi-\rho \rightarrow \pi+\rho$ & $\pi+\rho \rightarrow \frac{3 \pi}{2}-\rho$ & $\frac{3 \pi}{2}-\rho \rightarrow \frac{3 \pi}{2}+\rho$ & $\frac{3 \pi}{2}+\rho \rightarrow 2 \pi-\rho$ \\
\hline$R_{1}$ & $\mathrm{~L}$ & $\mathrm{M}$ & $\mathrm{M}$ & $\mathrm{M}$ & $\mathrm{M}$ & $\mathrm{M}$ & $\mathrm{M}$ \\
\hline$R_{2}$ & $\mathrm{M}$ & $\mathrm{M}$ & $\mathrm{L}$ & $\mathrm{M}$ & $\mathrm{M}$ & $\mathrm{M}$ & $\mathrm{M}$ \\
\hline$R_{3}$ & $\mathrm{M}$ & $\mathrm{M}$ & $\mathrm{M}$ & $\mathrm{M}$ & $\mathrm{M}$ & $\mathrm{M}$ & $\mathrm{M}$ \\
\hline$R_{4}$ & $\mathrm{M}$ & $\mathrm{M}$ & $\mathrm{M}$ & $\mathrm{M}$ & $\mathrm{L}$ & $\mathrm{M}$ & $\mathrm{M}$ & \\
\hline$\emptyset_{T}=\emptyset_{1}-\emptyset_{2}$ & $F(U+W) / 2$ & $F W$ & $F(U+W) / 2$ & $F W$ & $F(Q+W) / 2$ & $F W$ & $F(Q+W) / 2$ & $\mathrm{M}$ \\
\hline$\emptyset_{B}=\emptyset_{3}-\emptyset_{4}$ & $-F(Q+W) / 2$ & $-F W$ & $-F(Q+W) / 2$ & $-F W$ & $-F(U+W) / 2$ & $-F W$ & $-F(U+W) / 2$ & $-F W$ \\
\hline$\emptyset_{L}=\emptyset_{4}-\emptyset_{1}$ & $-F(U-Q) / 2$ & 0 & 0 & 0 & $F(U-Q) / 2$ & 0 & 0 & 0 \\
\hline$\emptyset_{R}=\emptyset_{2}-\emptyset_{3}$ & 0 & 0 & $-F(U-Q) / 2$ & 0 & 0 & 0 & $F(U-Q) / 2$ & 0 \\
\hline
\end{tabular}


Where :

$\mathrm{L}$ is the minimum radial air gap reluctance

$\mathrm{M}$ is the maximum radial air gap reluctance

$\mathrm{R}$ is the axial air gap reluctance

$U=\frac{2 R+M}{R L+R M+L M}$

$Q=\frac{2 R+L}{R L+R M+L M}$

$W=\frac{2 R+M}{R M+R M+M M}$

Integrating equation (6) into the fluxes waveforms given in table (1) leads to highly complicated wave form. Anyway, whatever the degree of complicity of the flux waveform, its local value still equal to the MMF multiplied by the air gap function $g^{-1}$ as given in equation (7). The equivalent air gap function can be approximated by Fourier series expansion as given in equation (8).

$$
\begin{aligned}
& \emptyset_{x}\left(\theta-\theta_{r m}\right)=\frac{1}{2} F(\theta) \cdot g_{x}^{-1}\left(\theta-\theta_{r m}\right) \\
& g_{x}^{-1}=A_{0}+\sum_{j=1}^{n} A_{j} \sin \left(j\left(\theta-\theta_{r m}\right)-\tau_{j}\right)
\end{aligned}
$$

Where:

$x \in\{T, B, L, R\}$ for top, bottom, left and right equivalent air gaps respectively.

$\mathrm{j}$ harmonic order.

$\tau_{j}$ phase angle of harmonic wave $\mathrm{j}$.

$A_{j} \quad$ Amplitude of harmonic wave $\mathrm{j}$.

The proposed plan to move forward is as follows

Derive an equation for the flux linked by each winding in the machine based on equation (6-8) using the conventional way.

Derive the values for Fourier series coefficients for each equivalent air gap function based on table (1).

Integrating the Fourier series coefficient with the flux linkage equations and evaluate the inductance formula.

Assume an arbitrary coil of $n_{b} / 2$ turns starts at an angle $\gamma$ where $0<\gamma<\alpha$. Taking in consideration that half of the end turns is folded upward and the other half is folded downward, the flux linking the top half coil $\left(\lambda_{1 T}\right)$ is the integration of the flux wave crossing the top air gap over half electric period as given in equation (9). 


$$
\begin{aligned}
\lambda_{1 T}=-\frac{n_{a} n_{b} i}{16} \int_{\gamma}^{\alpha}\left\{A_{0}+\sum_{j=1}^{\infty} A_{j} \sin \left(j\left(\theta-\theta_{r m}\right)-\tau_{j}\right)\right\} d \theta \\
+\frac{n_{a} n_{b} i}{16} \int_{\beta}^{\gamma+\pi}\left\{A_{0}+\sum_{j=1}^{\infty} A_{j} \sin \left(j\left(\theta-\theta_{r m}\right)-\tau_{j}\right)\right\} d \theta
\end{aligned}
$$

Equation (9) is integrated and rearranged with attention paid to the effect of odd/even harmonic order. The result is given in equation (10).

$$
=\frac{n_{n} n_{b} i}{16}\left\{A_{0}(2 \gamma+\pi-\beta-\alpha)+\sum_{j=2(e v e n)}^{\infty} \frac{-2 A_{j}}{j} \cos \left(j\left(\gamma-\theta_{r m}\right)-\tau_{j}\right)+\sum_{j=1}^{\infty} \frac{A_{j}}{j}\left\{\cos \left(j\left(\alpha-\theta_{r m}\right)-\tau_{j}\right)+\cos \left(j\left(\beta-\theta_{r m}\right)-\tau_{j}\right)\right\}\right\}
$$

As each phase consists of two adjacent coils shifted by one slot angle $(\Delta)$, The flux links the top half of the second coil of the phase in concern $\left(\lambda_{2 T}\right)$ can be obtained by substituting $\gamma+\beta-\alpha$ in place of $\gamma$ in equation (10). The flux links the top half of the phase winding $\left(\lambda_{T}\right)$ is the sum of $\left(\lambda_{1 T}\right)$ and $\left(\lambda_{2 T}\right)$ as given in equation (11).

$$
\begin{aligned}
\lambda_{T}=\frac{n_{a} n_{b} i}{16}\{ & A_{0}(4 \gamma+2 \pi-4 \alpha) \\
& +\sum_{j=2(\text { even })}^{\infty} \frac{-2 A_{j}}{j} \cos \left(j\left(\gamma-\theta_{r m}\right)-\tau_{j}\right) \\
& +\sum_{j=2(e v e n)}^{\infty} \frac{-2 A_{j}}{j} \cos \left(j\left(\gamma+\beta-\alpha-\theta_{r m}\right)-\tau_{j}\right) \\
& \left.+\sum_{j=1}^{\infty} \frac{2 A_{j}}{j} \cos \left(j\left(\alpha-\theta_{r m}\right)-\tau_{j}\right)+\sum_{j=1}^{\infty} \frac{2 A_{j}}{j} \cos \left(j\left(\beta-\theta_{r m}\right)-\tau_{j}\right)\right\}
\end{aligned}
$$

Similar approach can be used to evaluate the flux links the lower half of the phase winding in the same direction $\left(-\lambda_{B}\right)$. It could be noticed from table (1) that the air gap function of the bottom air gap is the same as that of the top air gap but shifted in phase by $\pi$ electrical degree. The odd harmonics will be inverted in sign. Equation (12) provides the waveform of the flux crossing the lower half of the first coil in the phase $\left(-\lambda_{1 B}\right)$. 


$$
\begin{aligned}
-\lambda_{1 B}=\frac{F(\theta)}{2} & . \frac{n_{b}}{4} \int_{\gamma}^{\gamma+\pi}\left\{A_{0}\right. \\
& -\sum_{j=\text { odd }} A_{j} \sin \left(j\left(\theta-\theta_{r m}\right)-\tau_{j}\right) \\
& \left.+\sum_{j=\text { even }} A_{j} \sin \left(j\left(\theta-\theta_{r m}\right)-\tau_{j}\right)\right\} d \theta
\end{aligned}
$$

The flux links the lower half of the second coil $\left(-\lambda_{2 B}\right)$ and the flux links the lower part of the phase $\left(-\lambda_{B}\right)$ are calculated using the same previous technique. The result is shown in equation (13).

$$
\begin{aligned}
-\lambda_{B}=\frac{n_{a} n_{b} i}{16}\left\{\begin{array}{l}
A_{0}(4 \gamma+2 \pi-4 \alpha) \\
+
\end{array}\right. & \sum_{j=2(\text { even })}^{\infty} \frac{-2 A_{j}}{j} \cos \left(j\left(\gamma-\theta_{r m}\right)-\tau_{j}\right) \\
& +\sum_{j=2(\text { even })}^{\infty} \frac{-2 A_{j}}{j} \cos \left(j\left(\gamma+\beta-\alpha-\theta_{r m}\right)-\tau_{j}\right) \\
& +\sum_{j=2(e v e n)}^{\infty} \frac{2 A_{j}}{j} \cos \left(j\left(\alpha-\theta_{r m}\right)-\tau_{j}\right)+\sum_{j=2(\text { even })}^{\infty} \frac{2 A_{j}}{j} \cos \left(j\left(\beta-\theta_{r m}\right)-\tau_{j}\right) \\
& +\sum_{j=1(\text { odd })}^{\infty} \frac{-2 A_{j}}{j} \cos \left(j\left(\alpha-\theta_{r m}\right)-\tau_{j}\right) \\
& \left.+\sum_{j=1(\text { odd })}^{\infty} \frac{-2 A_{j}}{j} \cos \left(j\left(\beta-\theta_{r m}\right)-\tau_{j}\right)\right\}
\end{aligned}
$$

It could be understood that some of the flux links the upper half of the phase downward $\left(\lambda_{T}\right)$ prefers the axial path while the rest $\left(-\lambda_{B}\right)$ continues downward and links the lower half of the phase. Hence, the total flux linkage the phase $\lambda$ is the sum of $\lambda_{T}$ and $-\lambda_{B}$ as given in equation (14). 


$$
\begin{aligned}
& \lambda=\frac{n_{a} n_{b} i}{4}\left\{A_{0}(2 \gamma+\pi-2 \alpha)+\sum_{j=\text { even }}^{\infty} \frac{-A_{j}}{j} \cos \left(j\left(\gamma-\theta_{r m}\right)-\tau_{j}\right)\right. \\
& \quad+\sum_{j=\text { even }}^{\infty} \frac{-A_{j}}{j} \cos \left(j\left(\gamma+\beta-\alpha-\theta_{r m}\right)-\tau_{j}\right) \\
&\left.+\sum_{j=\text { even }} \frac{A_{j}}{j} \cos \left(j\left(\alpha-\theta_{r m}\right)-\tau_{j}\right)+\sum_{j=\text { even }} \frac{A_{j}}{j} \cos \left(j\left(\beta-\theta_{r m}\right)-\tau_{j}\right)\right\}
\end{aligned}
$$

Equation (14) shows that the total phase to phase flux linkage contains only even harmonics. The next step in the analysis is the derivation of the amplitude and phase angle of each harmonic in the entire equivalent air gap functions. This could be done by direct implementation of Fourier analysis for the wave forms stated in table (1) where the result for the top and bottom air gaps is stated in equation (15).

$$
\begin{aligned}
& g_{T}^{-1} \\
& =\frac{4}{2 \pi}[\rho(U+Q)+(\pi W-2 \rho W)] \\
& +\sum_{j=1}^{\infty}\left\{\begin{array}{lr}
\frac{2}{\pi j}(Q-U) \sin (j \rho)\left[\sin \left(j\left(\theta-\theta_{r m}\right)\right)-\cos \left(j\left(\theta-\theta_{r m}\right)\right)\right] \mathrm{J}=1,5,9,13 \\
0 & \mathrm{~J}=2,6,10,14 \\
\frac{-2}{\pi j}(Q-U) \sin (j \rho)\left[\sin \left(j\left(\theta-\theta_{r m}\right)\right)-\cos \left(j\left(\theta-\theta_{r m}\right)\right)\right] & \mathrm{J}=3,7,11,15 \\
\frac{4}{\pi j}(Q+U-2 W) \sin (j \rho) \sin \left(j\left(\theta-\theta_{r m}\right)\right) & \mathrm{J}=4,8,12,16
\end{array}\right.
\end{aligned}
$$

Equation (15) confirms that the air gap function of the top and bottom air gaps does not contain harmonics of orders (2,6,10 etc.), while equation (14) tells that all odd harmonics vanishes in the resultant phase to phase flux linkage. It means that the remaining harmonics are the quadruple harmonics. It makes sense to consider only the fourth harmonics after merging equations (14 and 15), keeping in mind that the quadruple harmonics phase angle is zero as shown in equation (15). The mutual inductance among the stator phases reaches the form in equation (16).

$$
L_{\alpha \gamma}=\frac{n_{a} n_{b} i}{4}\left\{\begin{array}{c}
A_{0}(2 \gamma+\pi-2 \alpha) \\
+\frac{A_{4}}{4}\left\{\begin{array}{c}
{[-\cos (4 \gamma)-\cos (4(\gamma+\Delta))+\cos (4 \alpha)+\cos (4(\alpha+\Delta))] \cos \left(4 \theta_{r m}\right)} \\
+[-\sin (4 \gamma)-\sin 4(\gamma+\Delta)+\sin (4 \alpha)+\sin (4(\alpha+\Delta))] \sin \left(4 \theta_{r m}\right)
\end{array}\right\}
\end{array}\right\}
$$

Where :

$$
\begin{gathered}
A_{0}=\frac{4}{2 \pi}[\rho(U+Q)+(\pi W-2 \rho W)] \\
A_{4}=\frac{1}{\pi}(Q+U-2 W) \sin (j \rho)
\end{gathered}
$$

Equation (16) has been derived based on the assumption that $0<\gamma<\alpha$ and both of $\gamma$ 
and $\alpha$ are principle angles while these conditions are not valid for all mutual phase to phase combination. In fact the issue is the integration limits which should be varied to match each $\alpha$ $\gamma$ special location. The integration limit affects only the constant part of the inductance. Equation (17) provides general formula for the constant part of the phase to phase inductance $\left(\lambda_{0}\right)$.

$$
\lambda_{0}=\frac{n_{a} n_{b} i}{4} A_{0}(\pi-2|\varepsilon|)
$$

Where $: \mathrm{k}$ is constants

$$
\varepsilon=\gamma-\alpha \pm k \pi:-\pi<\varepsilon<\pi
$$

The armature self and phase to phase mutual inductance for machines with near sinusoidal and concentric windings are stated in table (2) in the first two columns.

\begin{tabular}{|c|c|c|c|c|}
\hline \multirow[t]{2}{*}{$\mathrm{L}$} & \multicolumn{3}{|c|}{ Square Air Gap } & \multirow{2}{*}{ Sinusoidal Air Gap } \\
\hline & Near sinusoidal winding $\left(\Delta=\frac{\pi}{6}\right)$ & Concentric winding $(\Delta=0)$ & Sinusoidal winding & \\
\hline $\begin{array}{l}\text { Armature self } \\
\text {-inductance }\end{array}$ & $\frac{n^{2} \pi A_{0}}{4}$ & $\frac{n^{2} \pi A_{0}}{4}$ & $n^{2} \pi A_{0}$ & $\frac{n^{2} \pi A_{0}}{4}$ \\
\hline$L_{A B}$ & $\frac{n^{2}}{4}\left[\frac{-\pi}{3} A_{0}+\frac{A_{4 \sqrt{3}}}{4} \sin \left(4 \theta_{r m}\right)\right]$ & $\frac{n^{2}}{4}\left[\frac{-\pi}{3} A_{0}+\frac{A_{4 \sqrt{3}}}{2} \sin \left(4 \theta_{r m}+\frac{\pi}{3}\right)\right]$ & $\frac{-n^{2} \pi A_{0}}{2}$ & $\frac{-n^{2} \pi A_{0}}{12}$ \\
\hline$L_{B C}$ & $\frac{n^{2}}{4}\left[\frac{-\pi}{3} A_{0}+\frac{A_{4 \sqrt{3}}}{4} \sin \left(4 \theta_{r m}+\frac{2 \pi}{3}\right)\right]$ & $\frac{n^{2}}{4}\left[\frac{-\pi}{3} A_{0}+\frac{A_{4 \sqrt{3}}}{2} \sin \left(4 \theta_{r m}+\pi\right)\right]$ & $\frac{-n^{2} \pi A_{0}}{2}$ & $\frac{-n^{2} \pi A_{0}}{12}$ \\
\hline$L_{C A}$ & $\frac{n^{2}}{4}\left[\frac{-\pi}{3} A_{0}+\frac{A_{4 \sqrt{3}}}{4} \sin \left(4 \theta_{r m}-\frac{2 \pi}{3}\right)\right]$ & $\frac{n^{2}}{4}\left[\frac{-\pi}{3} A_{0}+\frac{A_{4 \sqrt{3}}}{2} \sin \left(4 \theta_{r m}-\frac{\pi}{3}\right)\right]$ & $\frac{-n^{2} \pi A_{0}}{2}$ & $\frac{-n^{2} \pi A_{0}}{12}$ \\
\hline$L_{A L}$ & $1.93 n n_{f} C_{1} \cos \left(\theta_{r m}-\frac{\pi}{12}\right)$ & $n n_{f} C_{1} \cos \left(\theta_{r m}\right)$ & $n_{a} n_{f} \pi C_{1} \cos \left(\theta_{r m}-\frac{\pi}{2}\right)$ & $Z n n_{f} C_{1} \cos \left(\theta_{r m}-\sigma\right)$ \\
\hline$L_{A R}$ & $1.93 n n_{f} C_{1} \cos \left(\theta_{r m}+\frac{5 \pi}{12}\right)$ & $n n_{f} C_{1} \cos \left(\theta_{r m}+\frac{\pi}{2}\right)$ & $n_{a} n_{f} \pi C_{1} \cos \left(\theta_{r m}\right)$ & $Z n n_{f} C_{1} \cos \left(\theta_{r m}+\frac{\pi}{2}-\sigma\right)$ \\
\hline$L_{B L}$ & $1.093 n n_{f} C_{1} \cos \left(\theta_{r m}-\frac{17 \pi}{12}\right)$ & $n n_{f} C_{1} \cos \left(\theta_{r m}-\frac{4 \pi}{3}\right)$ & $n_{a} n_{f} \pi C_{1} \cos \left(\theta_{r m}-\frac{11 \pi}{6}\right)$ & $Z n n_{f} C_{1} \cos \left(\theta_{r m}-\frac{4 \pi}{3}-\sigma\right)$ \\
\hline$L_{B R}$ & $1.93 n n_{f} C_{1} \cos \left(\theta_{r m}-\frac{11 \pi}{12}\right)$ & $n n_{f} C_{1} \cos \left(\theta_{r m}-\frac{5 \pi}{6}\right)$ & $n_{a} n_{f} \pi C_{1} \cos \left(\theta_{r m}-\frac{4 \pi}{3}\right)$ & $Z n n_{f} C_{1} \cos \left(\theta_{r m}-\frac{5 \pi}{6}-\sigma\right)$ \\
\hline$L_{C L}$ & $1.93 n n_{f} C_{1} \cos \left(\theta_{r m}-\frac{3 \pi}{4}\right)$ & $n n_{f} C_{1} \cos \left(\theta_{r m}-\frac{2 \pi}{3}\right)$ & $n_{a} n_{f} \pi C_{1} \cos \left(\theta_{r m}-\frac{7 \pi}{6}\right)$ & $Z n n_{f} C_{1} \cos \left(\theta_{r m}-\frac{2 \pi}{3}-\sigma\right)$ \\
\hline$L_{C R}$ & $1.93 n n_{f} C_{1} \cos \left(\theta_{r m}-\frac{\pi}{4}\right)$ & $n n_{f} C_{1} \cos \left(\theta_{r m}-\frac{\pi}{6}\right)$ & $n_{a} n_{f} \pi C_{1} \cos \left(\theta_{r m}-\frac{2 \pi}{3}\right)$ & $Z n n_{f} C_{1} \cos \left(\theta_{r m}-\frac{\pi}{6}-\sigma\right)$ \\
\hline 1 self inductan & $L_{f f}$ & $L_{f f}$ & $L_{f f}$ & $L_{f f}$ \\
\hline
\end{tabular}

Table 2: inductances of the BSPRHM

$\mathrm{Z}=\{1.93,1, \pi\}, \sigma=\left\{\frac{\pi}{12}, 0, \frac{\pi}{2}\right\}$ for near sinusoidal, concentric and sinusoidal windings respectively.

The mutual inductance between the field and armature winding is evaluated by applying equations (7 and 8) to the axial fluxes $\emptyset_{L}$ and $\emptyset_{R}$ for the left and right hand side field coils respectively while using the MMF generated by the field coils. Then, integrating the results over a complete cycle as shown in equation (18 and 19) respectively.

$$
\begin{aligned}
\lambda_{L}=-\frac{n_{a} n_{f} i}{4} \int_{\beta+\pi}^{\alpha+2 \pi}\left\{C_{0}+\sum_{j=1} C_{j} \sin \left(j\left(\theta-\theta_{r m}\right)-T_{j}\right)\right\} d \theta & \\
& +\frac{n_{a} n_{f} i}{4} \int_{\beta}^{\alpha+\pi}\left\{C_{0}+\sum_{j=1} C_{j} \sin \left(j\left(\theta-\theta_{r m}\right)-T_{j}\right)\right\} d \theta
\end{aligned}
$$




$$
\begin{aligned}
\lambda_{R}=-\frac{n_{a} n_{f} i}{4} \int_{\beta+\pi}^{\alpha+2 \pi}\left\{C_{0}+\sum_{j=1} C_{j} \sin \left(j\left(\theta-\theta_{r m}-\frac{\pi}{2}\right)-T_{j}\right)\right\} d \theta \\
\quad=\frac{n_{a} n_{f} i}{4} \int_{\beta}^{\alpha+\pi}\left\{C_{0}+\sum_{j=1} C_{j} \sin \left(j\left(\theta-\theta_{r m}-\frac{\pi}{2}\right)-T_{j}\right)\right\} d \theta
\end{aligned}
$$

Where :

$n_{f}$ is the number of turns of the field windings

$C_{j}$ and $T_{j}$ are the amplitude and phase angle of the axial flux Jth harmonics

The armature to field mutual inductance is got by manipulating and rearranging of equation (18 and 19) as given in equations (20 and 21).

$$
\begin{gathered}
L_{\alpha L}=\frac{n_{a} n_{f}}{2} \sum_{j=o d d}\left\{\frac{C_{j}}{j} \cos \left(j\left(\alpha-\theta_{r m}\right)-T_{j}\right)+\frac{C_{j}}{j} \cos \left(j\left(\alpha+\Delta-\theta_{r m}\right)-T_{j}\right)\right\} \\
L_{\alpha R}=\frac{n_{a} n_{f}}{2} \sum_{j=o d d}\left\{\frac{C_{j}}{j} \cos \left(j\left(\alpha-\theta_{r m}-\frac{\pi}{2}\right)-T_{j}\right)\right. \\
\left.+\frac{C_{j}}{j} \cos \left(j\left(\alpha+\Delta-\theta_{r m}-\frac{\pi}{2}\right)-T_{j}\right)\right\}
\end{gathered}
$$

The amplitude and phase angle of the axial flux equivalent air gap function are obtained by direct implementation of Furrier analysis to the axial flux given in table (1) as shown in equation (22)

$$
\left\{\begin{array}{c}
C_{j}=\frac{4(U-Q)}{\pi j} \sin (j \rho) j \neq 0 \\
T_{j}=0 \\
C_{0}=0
\end{array}\right.
$$

Considering only the fundamental component, the armature to field mutual inductance for machines with near sinusoidal and concentric windings is stated in table (2) in the first two columns.

The field self-inductance value is constant as the magnetic pass seen by the field coil in this case is the same regard less of the rotor position as given in equation (23).

$$
L_{f f}=\frac{n_{f}^{2}}{\left[\frac{\rho L(\pi-\rho)}{M \pi(\pi-\rho)+\rho L \pi}+R\right]}
$$


The theoretical winding distribution is purely sinusoidal. The MMF for a sinusoidal distributed winding of $n_{a}$ coil is stated at equation (24) [5].

$F(\theta)=2 n_{a} i \cos (\theta-\alpha)$

The inductance matrix of a machine that has sinusoidal distributed winding can be derived by replacing equation (6) by equation (24) and repeat the previous analysis. The result is shown at equations (25 to 27 ).

$$
\begin{aligned}
& L_{\alpha \gamma}=A_{0} n_{b} n_{a} \pi \cos (\gamma-\alpha) \\
& L_{\alpha L}=n_{a} n_{f} \pi C_{1} \cos \left(\theta_{r m}-\frac{\pi}{2}-\alpha+\tau\right) \\
& L_{\alpha R}=n_{a} n_{f} \pi C_{1} \cos \left(\theta_{r m}-\alpha+\tau\right)
\end{aligned}
$$

It is obvious from equations ( 25 to 27 ) that the sinusoidal windings eliminate the harmonic contents from the entire machine inductances. Another way to eliminate the harmonics is designing a machine with pure sinusoidal air gap function.

The inductances for machines with sinusoidal windings and sinusoidal air gap function are stated in table (2) in the last two columns respectively.

Once the inductance matrix is determined, the classical machine model can be used with the BSPRHM as given in equations (28-31).

$$
\begin{aligned}
& \qquad V=R I+\frac{d \lambda}{d t} \\
& \lambda=L I \\
& \text { Torque }=\frac{1}{2} I^{T} \frac{d L}{\theta_{r m}} I \\
& \text { Torque }- \text { Load torque }=J \frac{d W_{r}}{d t}+B W_{r}
\end{aligned}
$$

\section{3- Experimental Verses Simulation}

A four poles prototype for the proposed BSPRHM is built based on a square air gap design with the near sinusoidal windings shown in fig. 5. First an experiment is carried out to determine the values of the inductance matrix elements of the BSPRHM. An alternating voltage source of frequency $50 \mathrm{~Hz}$ is used to energize only one of the machine five windings. The RMS current of the energized winding and the RMS voltage across each of the entire windings are measured and recorded. The phase angle between the current in the energized phase and each of the entire windings voltages is monitored on an oscilloscope. If the current leads the voltage, the associated inductance is considered negative. And the inductance is considered positive if the current lags the voltage. The magnitude of the inductance is calculated using equation (32).

$$
L_{x y}\left(\theta_{r m}\right)=\frac{V_{x}\left(\theta_{r m}\right)}{2 \pi f \cdot I_{y}\left(\theta_{r m}\right)}
$$


$x, y \in\{A, B, C, L, R\}$

$f$ is the supply frequency

An encoder attached to the BSPRHM shaft is used to the measure the rotor position.

The rotor position $\theta_{r m}$ is scanned along a complete shaft revolution in steps of 10 degrees and the inductances magnitude and sign are re-measured in each step. Then, the power supply is transferred to energize another phase and the same previous procedure is repeated. The row data collected from the experiment is fed to the matlab software to calculate and plot the 25 inductances values against the rotor position. The result is shown in fig. 5. Analysis of the results shown in fig. 5 leads to a number of observations. The wave form of the mutual inductance between the armature phases contains eight peaks within one mechanical revolution. As the prototype machine has four poles, the existence of the forth harmonic in these inductances is proved. The practical values of the phase angles of the entire inductances profile shown in fig. 5 is the same as its theoretical values given in the equations in the first column of table (2). Other points of similarity between the practical and theoretical inductance profile are the negative sign of the average value of the mutual inductances between stator phases, the constant positive value of the entire windings self-inductances and the two peak per revolution sinusoidal waveform of the mutual inductance between stator phases and field coils which indicates dominant fundamental component for four poles machine. The close to zero value of the field to field mutual inductance indicates small leakage flux through the nonmagnetic separator in the practical machine. The theoretical ratio between the mutual inductance between stator phases and the stator phases' self-inductance is one third while the practical value is less than one third which indicates leakage inductance in the stator phases. The previous analysis shows great similarity between the theoretical and practical inductances profile which proves the accuracy of mathematical derivation. The amplitude and mean values of the entire inductances is got from fig. 5. The odd data like the notch appears in the right field to armature phase A wave form is omitted. Also an average value is considered for the amplitude and mean of similar inductances waveforms. The resulting inductance matrix of the proto type is depicted in equation (33) 


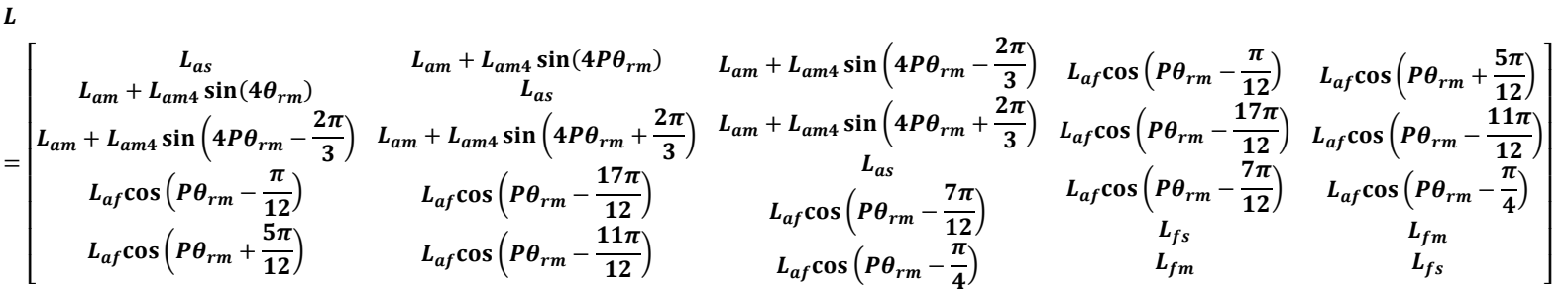

Where : $\left\{L_{a s}, L_{a m}, L_{a m 4}, L_{a f}, L_{f s}, L_{f m}, P\right\}=\{775,-170,46.5,153,787,0.85,2\}$ respectively.
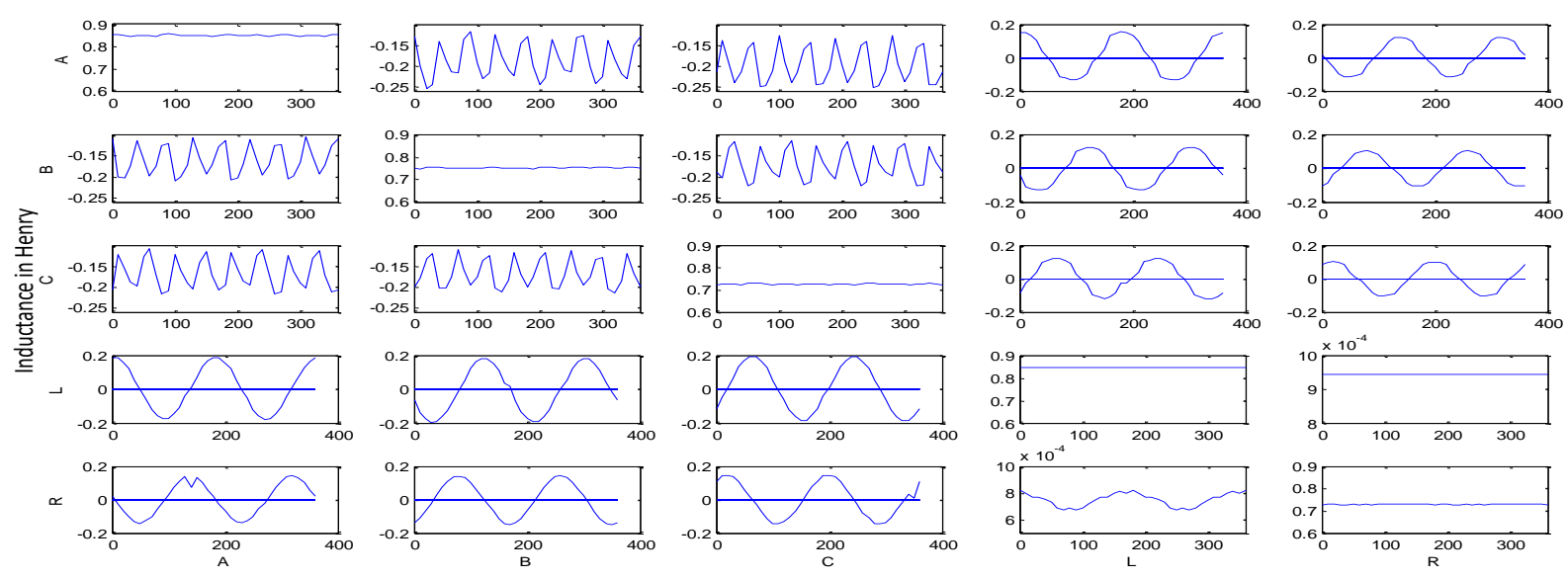

Mechanical Rotor Position in Degree

Fig. 5: BSPRHM inductances variation

Another practical experiment is conducted to verify the relation between the armature frequency, field frequency and rotor speed given in equation (1). The BSPRHM is run in the generator mode and the prime mover speed is changed in steps. At each step the field frequency is varied while monitoring the open circuit armature voltage frequency until the armature frequency reached $50 \mathrm{~Hz}$. The practical values of the field frequency and rotor speed are recorded and plotted. On the same graph the theoretical and simulation results are plotted. The theoretical values are evaluated using equation (1) for armature frequency of 50 and $-50 \mathrm{~Hz}$. The simulation is done based on equations (28, 29 and 33). During the simulation the rotor speed is varied in steps and the field frequency is scanned until the armature frequency value reached $50 \mathrm{~Hz}$. The simulation is repeated for armature frequency of $-50 \mathrm{~Hz}$. Fig. 6 compares among the theoretical, simulation and practical result and provides additional proof for the validity of the proposed inductance matrix. 


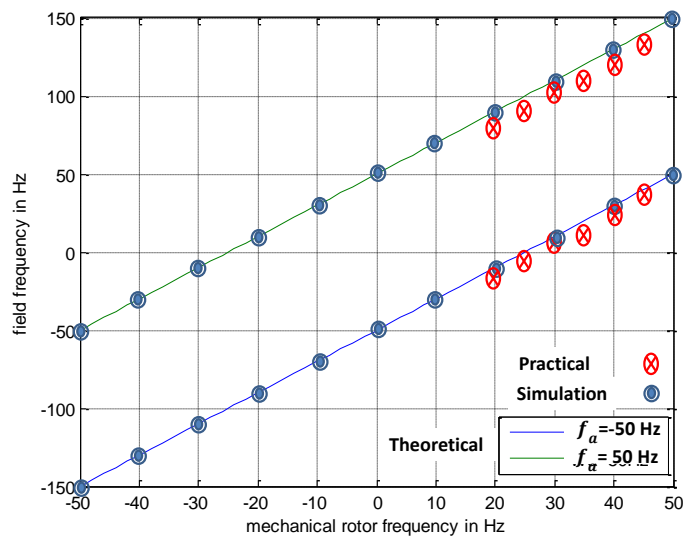

Fig. 6: Relation among rotor speed, field frequency and armature frequency of the BSPRHM.

\section{4- Conclusion}

This work presents the mathematical derivation of the BSPRHM inductance matrix. The proposed inductance matrix makes it possible to simulate the BSPRHM based on the classical machine mathematical model. A prototype is built and used to verify the validity of the proposed inductance matrix. The great similarity among the theoretical, simulation and practical results proves the accuracy of the proposed inductance matrix of the BSPRHM.

\section{References}

[1]Mohamed Magdy, Hany M. Hasanien, Hussien F. Soliman, and Elhussien A. Mahmoud, "On-Line ANN based Controller for Improving Transient Response of Grid-Connected DFIG-Driven by wind Turbine", International Journal of Recent Trend in Engineering \& Research, IJRTER, April 2018, Vol. 4, Issue 4, PP. 230-245

[2]Ramtin Sadeghi, Sayed M. Madani and Mohammad Ataei, “A New Smooth Synchronization of Brushless Doubly-Fed Induction Generator by Applying a Proposed Machine Model", IEEE Transaction on Sustainable Energy, Jan 2018, Vol. 9, Issue 1, PP.371-380.

[3] Elhussien A. Mahmoud, M. Nasrallah, Hussien F. Soliman and Hany M. Hasanien, “ Fractional Order PI controller Based on Hill Climbing Technique for Improving MPPT of the BDF-RG Driven by Wind Turbine:, 19th international middle-east power system conference (MEPCON'19, December 2017.

[4] Elhussien A. Mahmoud, "Brushless Slip Power Recovery Version of a Homopolar Inductor Machine", world journal of engineering research and technology. WJERT. 2018, Vol. 4, Issue 3, PP. 234-248.

[5] Robert E. Betz and Milutin Jovanovic. "Introduction to Brushless Doubly Fed Reluctance Machines- The Basic Equations”, March 2012, technical report, ftp://vcs2.newcastle.edu.au/papers/BDFRMRev.pdf 